\title{
PATTERN OF ELEVATIONAL DISTRIBUTION AND RICHNESS OF NON VOLANT MAMMALS IN ITATIAIA NATIONAL PARK AND ITS SURROUNDINGS, IN SOUTHEASTERN BRAZIL
}

\author{
GEISE, L., ${ }^{1}$ PEREIRA, L. G., ${ }^{2}$ BOSSI, D. E. P. ${ }^{3}$ and BERGALLO, H. G. ${ }^{4}$ \\ ${ }^{1}$ Departamento de Zoologia, Instituto de Biologia, Universidade do Estado do Rio de Janeiro, \\ Rua São Francisco Xavier, 524, CEP 20559-900, Rio de Janeiro, RJ, Brazil \\ ${ }^{2}$ Departamento de Genética, CCS, Universidade Federal do Rio de Janeiro, \\ Ilha do Fundão, Rio de Janeiro, RJ, Brazil \\ ${ }^{3}$ Departamento de Parasitologia, Instituto de Biologia, Universidade Estadual de Campinas, \\ C.P. 6109, CEP 13083-970, Campinas, SP, Brazil \\ ${ }^{4}$ Departamento de Ecologia, Instituto de Biologia, Universidade do Estado do Rio de Janeiro, \\ Rua São Francisco Xavier, 524, CEP 20559-900, Rio de Janeiro, RJ, Brazil \\ Correspondence to: Lena Geise, Departamento de Zoologia, Instituto de Biologia, Universidade do Estado do Rio de \\ Janeiro, Rua São Francisco Xavier, 524, CEP 20559-900, Rio de Janeiro, RJ, Brazil \\ Received November 8, 2002 - Accepted July 21, 2003 - Distributed August 31, 2004 \\ (With 3 figures)
}

\begin{abstract}
Itatiaia National Park (PNI) and its surroundings present a unique fauna due to different forest formations with well-defined climatic and vegetation bands. The Itatiaia massif has four vegetation types that follow an altitudinal gradient: lower montane forest, montane forest, upper montane forest, and Campos de Altitude. Hence, this region is ideal for studying geographical variation in biological diversity. The main objectives of this study were to report on nonvolant mammal species known to occur in Itatiaia National Park and its surroundings and to determine if their distributional pattern is related to elevation. A review of the literature and a complete survey of specimens deposited in museums, as well as small-mammal trapping were carried out in order to obtain a complete record of the species from the region. Precise locality data were obtained for all specimens recorded, allowing the inclusion of each collected or observed individual in an altitude and vegetational class. We made a direct ordination gradient of marsupial, primate, and rodent species abundance with the altitude. Sixty-nine mammal species were collected or reported for the Itatiaia massif, belonging to seven orders and 20 families. Of these, 33 species $(47.8 \%)$ are included in the official list of threatened or believed-tobe threatened species in Rio de Janeiro State. The orders Rodentia, Carnivora, and Didelphimorphia had the highest species richness, with 25,14 , and 13 species respectively. When species were grouped according to the vegetation, 16 species occured in the lower montane, 56 in the montane forest, five in the upper montane, and 21 in the high-altitude fields (Campos de Altitude). The communities of marsupials, primates, and rodents have an ordination pattern related to the altitude. Species richness was higher between $500 \mathrm{~m}$ and $1,500 \mathrm{~m}$ above sea level in montane forest, which is in agreement with recent studies showing that species richness can reach its maximum at mid-elevations.
\end{abstract}

Key words: species composition, altitudinal gradient, inventories, vegetational types, Atlantic Forest.

\section{RESUMO}

Padrão de distribuição altitudinal e riqueza de mamíferos não-voadores no Parque Nacional do Itatiaia e seu entorno, no Sudeste do Brasil

O Parque Nacional do Itatiaia e seu entorno apresenta fauna peculiar em razão de diferentes formações vegetais, consistindo em faixas definidas de clima e de vegetação. O maciço do Itatiaia tem quatro 
tipos de vegetação que seguem um gradiente altitudinal: Floresta Submontana, Floresta Montana, Floresta Alto-Montana e Campos de Altitude. Por isso, essa região é ideal para estudar variação geográfica na diversidade biológica. O principal objetivo deste estudo foi relatar as espécies de mamíferos não-voadores conhecidas para o Parque Nacional do Itatiaia e seu entorno e determinar se elas apresentam padrão de distribuição altitudinal. Foram realizados revisão completa da literatura e levantamento de espécimes depositados em museus, bem como esforços de captura de pequenos mamíferos, a fim de obter o levantamento completo das espécies da região. Dados precisos sobre as localidades foram obtidos para todos os espécimes levantados, permitindo a colocação de cada indivíduo coletado ou observado dentro de uma classe de altitude e de vegetação. Foi elaborado gradiente de ordenação direto da abundância de espécies de marsupial, de primatas e de roedores com a altitude. Foram coletadas ou relatadas 69 espécies de mamíferos para o maciço do Itatiaia, pertencendo a 7 ordens e 20 famílias. Dessas, 33 espécies (47,8\%) estão incluídas na lista oficial de espécies ameaçadas de extinção, ou presumivelmente ameaçadas, do Estado do Rio de Janeiro. As ordens Rodentia, Carnivora e Didelphimorphia apresentaram as mais altas riquezas de espécies, com 25, 14 e 13 espécies, respectivamente. Quando agrupadas de acordo com a vegetação, 16 espécies ocorreram na Floresta Submontana, 56 ocorreram na Floresta Montana, 5, na Floresta Alto-Montana e 21, nos Campos de Altitude. As comunidades de marsupiais, primatas e roedores têm padrão de ordenação relacionado com a altitude. A riqueza de espécies foi maior entre $500 \mathrm{~m}$ e $1.500 \mathrm{~m}$, na Floresta Montana, o que está de acordo com estudos recentes que mostram que a riqueza de espécies alcança seu valor máximo em elevações medianas.

Palavras-chave: composição de espécies, gradiente altitudinal, inventário, tipos de vegetação, Mata Atlântica.

\section{INTRODUCTION}

Rio de Janeiro State has been largely ignored as far as the study of its mammals is concerned. In the only extensive study of this type (Tribe, 1987), 77 nonvolant mammal species (excluding Cetacea and considering only native species) were reported. Pereira et al. (2001), also studying nonvolant terrestrial mammals, listed 43 species from the southern coastal region of Rio de Janeiro State while Bueno (1998) found 21 species in Tijuca National Park. Since the beginning of last century, Itatiaia National Park (PNI) has attracted special attention because of its fauna (Ávila-Pires \& Gouvêa, 1977; Holt, 1928; Pinto, $1951,1954)$ and the range of different forest formations found there, identified for the first time by Ule (1896). In 1965, Segadas-Viana \& Dau showed that the Itatiaia massif presents zones and well-defined climatic and vegetation bands. According to Ururahy et al. (1983), the Itatiaia massif has four vegetation types that follow an altitudinal gradient: lower montane forest (from 400 to $499 \mathrm{~m}$ in the study area); montane forest (from 500 to $1,499 \mathrm{~m}$ ); upper montane forest (from 1,500 to 1,999 m), and high-altitude fields (more than 2,000 m). Recently, Safford (1999) compared the high-altitude fields of some localities in sou- theastern Brazil (Itatiaia, Serra da Mantiqueira, Serra do Caparaó, Serra dos Órgãos, and Serra do Mar) with the páramos of the Andes, and showed a floristic similarity that extends to climate and soil.

The geographic localization of Itatiaia National Park, between two large urban centers, and the fact that the high-altitude fields are islands in a varied and extensive landscape, characterizes this region as a nucleus of the Atlantic Forest Biosphere Reserve, one of the biggest conservation units in the world (Conservation International of Brasil, 2000).

The PNI and its surrounding provide an ideal opportunity for studying geographical variation inbiological diversity. Studies of animal distribution along an altitudinal gradient provide useful information on their ecology, habitat preferences, and species replacement (Goodman et al., 1996). The four vegetation types described for the Itatiaia massif present distinct soil and floristic composition (Ururahy et al., 1983). Hence, each species of the mammal fauna is expected to be more strongly associated with one or another type of vegetation.

Besides testing the hypothesis that species from some orders (Didelphimorphia, Primates, and Rodentia) follow a pattern of distribution associated with altitude, we also have made an up-to-date list of 
nonvolant mammal species known to occur in PNI and its surroundings, along with some karyological information as well as conservation status.

\section{MATERIAL AND METHODS}

The field work aimed to increase the number of localities studied in the area and to provide material for correct specific identification (Rodentia, Sigmodontinae). We used banana, manioc, and peanut butter as bait in Sherman live traps. These were set differently depending on development of the research, topography, and the presence of trees, in which they were set at up to $7 \mathrm{~m}$ from the ground. The traps were generally placed in lines $c a .5$ to $20 \mathrm{~m}$ apart. Each line had from 10 to 25 traps, with none or a minimum of five arboreal traps, for a total of 100 traps open at least during three nights. Field work was carried out during the following periods: 12/29/95-1/2/96, 2/9/97-2/12/97, and 6/13/97-6/17/ 97 in Brejo da Lapa; 7/17/97-7/19/97 and 9/29/00$10 / 2 / 00$ in Serrinha do Alambari for trapping small non-volant mammals; and 8/20/99-8/23/99, 10/31/ $99-11 / 3 / 99$, and $2 / 1 / 00-2 / 4 / 00$ for direct observations of large mammals and for trapping small mammals at Piscina Maromba.

A review of the literature and a complete survey of specimens deposited in the Museu Nacional, Rio de Janeiro (MN-UFRJ), Museu de Zoologia da Universidade de São Paulo (MZUSP), and Museu do Parque Nacional de Itatiaia were carried out.

Field work was done with a permit issued by the Instituto Brasileiro do Meio Ambiente e dos Recursos Naturais Renováveis (IBAMA), \# 025/99, to H. G. Bergallo and L. Geise, and \# 02001.005474/ 98-96 to D. E. P. Bossi. After the complete analysis, specimens (skull, partial skeleton, and skin) will be deposited in the MN-UFRJ.

Precise locality data were obtained from the United States Board on Geographical Names - Brasil (1963), maps (Instituto Brasileiro de Geografia e Estatística - IBGE, scale 1:25,000 and 1:50,000), and during field work with a GPS and an altimeter. Vegetation types follow Ururahy et al. (1983).

Specimens were identified to the species level by comparison with voucher specimens deposited in museums, and by using morphological (skin and skull) descriptions available for Didelphimorphia (Emmons \& Feer, 1997; Lange \& Jablonski, 1998; Mustrangi \& Patton, 1997); Xenarthra and Carnivora
(Emmons \& Feer, 1997; Eisenberg \& Redford, 1999); Primates (Auricchio, 1995; Emmons \& Feer, 1997; Vivo, 1991); and Rodentia (Emmons \& Feer, 1997; Geise, 1995; Oliveira, 1998; Tribe, 1996; Weksler, 1996). Specific names followed Wilson \& Reeder (1993) in the absence of a more recent account. We adopted the name Cebus nigritus as a valid species, following Silva-Jr. (2001). The conservation status of each species is according to Bergallo et al. (2000). Finally, we considered as small mammals those species weighing up to $5 \mathrm{~kg}$ (Fleming, 1979).

When species identification was not possible by morphological comparison only, karyological data were used. Chromosomes were obtained from bone marrow cultures with cell cultures in MEM Dulbecco's medium (Ford \& Hamerton, 1956) with some modifications. Karyological data (diploid number $=2 \mathrm{n}$, fundamental number $=\mathrm{FN}$ ) for rodents of the subfamily Sigmodontinae (Muridae) are given according to Geise (1995) and Geise et al. (1998).

We made a direct ordination gradient of marsupial, primate, and rodent species abundance with altitude, since these orders were more widely reported in this study, by visualization, trapping, and voucher specimens. Direct gradient analysis focuses on response curves of species with respect to a complex of environment variables that gradually change in geographic space (Ter Braak \& Looman, 1995). Each species has its own response to the environmental gradient, and most of them have one optimum along the gradient and decline to either side (Gauch, 1982).

The record of each collected or observed individual was placed in an altitude class, each of which comprised $100 \mathrm{~m}$. The first class comprised altitudes between 400 and $499 \mathrm{~m}$ above sea level (a.s.1.); the second one, 500 and $599 \mathrm{~m}$ a.s.l.; and so on, up to the last, from 2,200 to 2,499 $\mathrm{m}$ a.s.l., representing two classes joined together, as few specimens were collected above 2,399 $\mathrm{m}$ a.s.l. To find the position of the species in the altitude gradient, the mean altitude reported for each species was calculated and the species ranked from the lowest to the highest mean. As capture results differed between altitudes, capture frequencies of each species in the altitude classes were transformed into proportions.

\section{RESULTS}

Specimens captured and karyotyped are indicated by $\leftrightarrow$. The $\diamond$ indicates visualizations without 
collecting numbers. Specimens listed with other symbols refer to records from: * Ávila-Pires \& Gouvêa (1977), \# Miranda-Ribeiro (1905), and • Oliveira (1998). Acronyms for institutions are: MN (Museu Nacional/UFRJ); MZUSP (Museu de Zoologia da Universidade de São Paulo); PNI (Museu do Parque Nacional de Itatiaia); AMNH (American Museum of Natural History); BMNH (British Museum of Natural History); and MCZ (Museum of Comparative Zoology, Harvard). Other prefixes correspond to field numbers of: LF (L. Flamarion); HGB, HGB-DB, and HGB-FMH (H.G. Bergallo) and LG (L. Geise). To avoid repetition of coordinates and altitude data, only the locality name is given after the first reference to a locality. The symbol for males is $\phi^{x}$; for females, $q$; and when sex was indeterminate, I. The conservation status (CS) is given when species are on the official list of threatened or believed-to-be threatened species in Rio de Janeiro State (Bergallo et al., 2000).

\section{Order Didelphimorphia}

Family Didelphidae

Caluromys philander (Linnaeus, 1758) Itatiaia, sede do Parque Nacional do Itatiaia (Sede

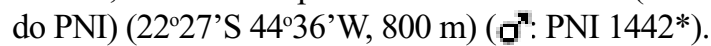

Didelphis aurita Wied-Neuwied, 1826 -

Resende, Serrinha do Alambari (2222'S 443' W, 850 m) (»); Itatiaia, site of PNI (800) ( $\sigma^{\star}:$ PNI 72*, 190*), site of PNI $(830 \mathrm{~m})\left(\sigma^{x}\right.$ : PNI $\left.770^{*}\right)$, site of PNI (850 m) ( $\sigma^{\pi}$ : PNI 1241*; $q$ : PNI 414*).

Lutreolina crassicaudata (Desmarest, 1804) Itatiaia, Maromba (according to Ávila-Pires and Gouvêa (1977), specimens were collected but not preserved). CS: threatened/critically endangered.

Marmosa murina (Linnaeus, 1758) - Itatiaia,

Rio Campo Belo (22²6'S 443'' W, 920 m) (I: MN $\left.1268^{*}\right)$; site of PNI (800 m) (\#, specimen (fur and skull) for this register was not found by us in any visited museum).

Marmosops incanus (Lund, 1840) - Resende, Serrinha do Alambari ( $\sigma^{x}$ : HGB-FMH 02, 9 : MN

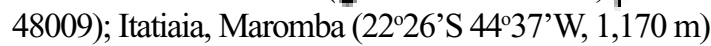
(I: HGB-DB 26), Penedo (22²3'S 443'' 500 m) (I: MZUSP 29172).

Marmosops paulensis Tate, 1931 - site of PNI (830 m) ( $\sigma^{x}$ : PNI 1036* - skin; PNI 1040* - skull, both numbers refer to the same individual). CS: believed threatened.

Micoureus travassosi (Thomas, 1920) Itatiaia, sede do PNI (800 m) ( $\sigma^{x}:$ PNI 46 - data from voucher cards), Macieiras (no coordinate data, 1,830 m) ( $\sigma^{x}$ : PNI $768^{*}$ - same number for a specimen of Philander frenatus).

Monodelphis dimidiata (Wagner, 1847) Itatiaia, site of PNI (815 m) ( $\sigma^{\star}:$ PNI $311^{*}, q$ : PNI 430*); site of PNI ( $850 \mathrm{~m}$ ) (Male: PNI 935); Maromba (900 m) I $\emptyset^{\pi}$ : PNI 1041*); Maromba-Mauá (1,200 m) (I: PNI 623*), Rio Campo Belo (920 m) (I: MN 1311).

Monodelphis americana (Müller, 1776) - Itatiaia, site of PNI (815 m) ( $\sigma^{x}:$ PNI 423*); site of PNI (830 m) (I: PNI 431); site of PNI (850 m) (\$: MZUSP 11695); Maromba (1170 m) ( $0^{x}:$ HGB-DB 35).

Monodelphis scalops (Thomas, 1888) - Itatiaia, site of PNI (815 m) ( $\sigma^{\star}$ : PNI 312*). CS: believed thereatened.

Monodelphis theresa Thomas, 1921 collected in the PNI by E. Gouvea, no specific locality cited (I: MZUSP 11695). CS: threatened/ probably extinct.

Philander frenatus Olfers, 1818 - Resende, Serrinha do Alambari ( $\diamond)$; Itatiaia, Penedo $\left(\sigma^{x}\right.$ : MZUSP 29209), site of PNI (550 m) (\$: AMNH $\left.61541^{*}\right)$, site of PNI (800 m) (Q: MN 1195*, PNI $193 *)$; site of PNI (830 m) (\#) (ơ': PNI 1034*, \%: PNI 1027*, MN 26618); Macieiras (1,830 m) (o': PNI 48* same number for a specimen of Sylvilagus brasiliensis, MZUSP 7424, 7425, I: PNI 768 - same number for a specimen of Micoureus travassosi); Brejo da Lapa (2223'S 4443'W, 2100 m) (Male: MN 48043); Mauá (234ㅇ'S 46²7’ W, 764 m) ( $0^{\star}: \mathrm{MN}$ 27833, 9 : MN 26783, I: MN 26799).

Thylamys velutinus (Wagner, 1842) - Resende, Serrinha do Alambari (\&: HGB-FMH 01).

\section{Order Xenarthra}

Family Bradypodidae

Bradypus variegatus Schinz, 1825 - Itatiaia, Maromba (lote 29) (1,000 m) (d': PNI 1449 - data from voucher cards), Maromba, lote 41 (1,200 m) (Q: PNI $146^{*}$ - data from voucher cards), Pinheiro Grande (lote 21) (no coordinate data, $850 \mathrm{~m}$ ) ( $\sigma^{x}$ : PNI 1359 - data from voucher cards).

Family Dasypodidae

Cabassous tatouay (Desmarest, 1804) Itatiaia, Macieiras $(1,830 \mathrm{~m})\left(\sigma^{x}:\right.$ PNI $55^{*}$ - same number for a specimen of Alouata fusca). CS: believed threatened.

Dasypus novemcinctus Linnaeus, 1758 Itatiaia, site of PNI (800 m) ( $\sigma^{\star}$ : PNI 864*, 1168* - data from voucher cards). 
Dasypus septemcinctus Linnaeus, 1758 Itatiaia, site of PNI (830 m) ( $\sigma^{x}$ : PNI 1048*), Maromba $(1,100 \mathrm{~m})\left(0^{x}\right.$ : PNI $\left.70^{*}\right)$. CS: believed threatened.

Family Myrmecophagidae

Myrmecophaga tridactyla Linnaeus, 1758 Itatiaia, Vale do Paraíba (no coordinate data, $450 \mathrm{~m}$ ) (I: PNI 816 - data from voucher cards). CS: threatened/probably extinct.

Tamandua tetradactyla (Linnaeus, 1758) Itatiaia, Vale do Paraíba (no coordinate data, $450 \mathrm{~m}$ ) (I: PNI 216*), site of PNI (800 m) (ơ : PNI 82*).

\section{Order Primates}

Family Callithrichidae

Callithrix aurita (É. Geoffroy, 1812) - Itatiaia (22029'S 4433'W, 400 m) (ỡ : MN 2828*; I: MN 2818*, 2819*); Casunga (no coordinate data, $750 \mathrm{~m}$ ) (ÁvilaPires \& Gouvêa, 1977); Santana (right margin of Paraíba River) ( $\sigma^{x}$ : PNI 1425a). CS: threatened/ vulnerable.

Callithrix penicillata (É. Geoffroy, 1812) Itatiaia (no locality data) (I: MN 2799); Itaboca, Santa Rita de Jacutinga (22 $04^{\prime}$ 'S $44^{\circ} 04^{\prime} \mathrm{W}, 790$ m) (I: MCZ 1433, 1824).

Family Cebidae

Alouatta fusca (É. Geoffroy, 1812) - Itatiaia, Hotel Granja Agulhas Negras (lote 92) (22²6'S

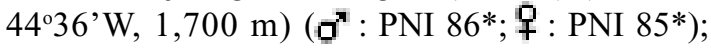
Maromba (1,100 m) (I: PNI 55 - same number for a specimen of Cabassous tatouay). CS: believed threatened.

Brachyteles arachnoides (É. Geoffroy, 1806) - Itatiaia, (22.29'S 44.33'W, 400 m) ( $\sigma^{x}:$ MZUSP 9962); PNI (no locality data, 1,000 m) (»); Maromba (Picadão) (1,300 m) (đ*: PNI 96*,, : PNI 95*, I: PNI 94*); Cachoeira da Maromba Road (22²6'S 44³7'W, 1,305 m) (»). CS: threatened/critically endangered. Callicebus nigrifrons (Spix, 1823) - Resende,

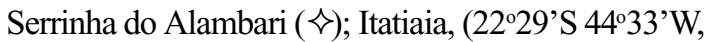
400 m) (I: MZUSP 7430); site of PNI (750 m) (đđ: PNI 1460, 1461); Rio Campo Belo, lote 27 (920 m) ( $0^{\star}$ PNI 75*, 9 : PNI 77*, I: PNI 57, 76*); Hotel Granja Agulhas Negras (lote 90) (1,150 m) (I: PNI 620*); Macieiras $(1,720 \mathrm{~m})$ (ㅇ: AMNH 61539*); Maromba (lote 29) $(1,100 \mathrm{~m})\left(\sigma^{\pi}:\right.$ PNI 1450, MZUSP 7426, 7429; \&: PNI 1459, MZUSP 7427, 7428, 19548); Chevap-funil (no locality data) ( $\sigma^{\pi}:$ MN 25897). CS: threatened/ vulnerable.
Cebus nigritus (Goldfuss, 1809) - Itatiaia, Sede do PNI (750 m) (\$: PNI 1466, 1467), site of PNI (800 m) ( $\sigma^{\star}:$ MN21171), Maromba, lot 96(1,100 m)( ( $:$ PNI 97*), Maromba (lot 41) (1,220 m) ( ( ${ }^{\pi}$ PNI 224*), Maromba (lot 41) $(1,250 \mathrm{~m})\left(\sigma^{\pi}:\right.$ PNI $\left.220^{*}\right)$.

\section{Order Carnivora}

Family Canidae

Cerdocyon thous (Linnaeus, 1766) - Itatiaia, road to Itamonte (BR 354) between kilometers 2021 (2229’S 4440’W, 500 m) (ㅇ: MN 42800); Vale do Paraíba (no coordinate data, $400 \mathrm{~m}$ ) ( o ${ }^{\star}:$ PNI 44*, 84* - same number for a specimen of Chrysocyon brachyurus, 187*, 753, 769*, 1172*, I: PNI 39); Campos do Itatiaia (22.23'S 44。40'W, 2,450 m) $\sigma^{x}$ : PNI 198*).

Chrysocyon brachyurus (Illiger, 1815)Itatiaia, Vale do Paraíba ( $\sigma^{*}$ : PNI 84 - data from voucher cards - same number for a specimen of Cerdocyon thous), Campos do Itatiaia $(2,400 \mathrm{~m})$ (I: PNI 714*). CS: believed threatened.

Family Felidae

Herpailurus yaguarondi (Lacépède, 1809) Itatiaia, Brejo da Lapa (22²1'S 44⒋' 'W, 2,125 m) $(\diamond)$.

Leopardus pardalis (Linnaeus, 1758) - Itatiaia, site of PNI $(800 \mathrm{~m})\left(\sigma^{\pi}\right.$ : PNI $\left.805^{*}\right)$. CS: threatened/ vulnerable.

Leopardus tigrinus (Schreber, 1775) - Itatiaia, Vale do Paraíba (400 m) (I: PNI 32*), sede do PNI (970 m) ( $\sigma^{\pi}$ : PNI 33*); Sítio Pitumendaba (22 $26^{\circ} \mathrm{S}$ $\left.44^{\circ} 37^{\prime} \mathrm{W}, 980 \mathrm{~m}\right)\left(\sigma^{x}:\right.$ PNI $1003^{*}$ - data from voucher cards); Recreio Maromba (22 $26^{\prime} \mathrm{S}$ $44^{\circ} 37^{\prime} \mathrm{W}, 850 \mathrm{~m}$ ) ( 9 : PNI 1441 - data from voucher cards); Hotel Alsene (22²2'S 4442'W, 2,290 m) (I: LG 87). CS: believed threatened.

Leopardus wiedii (Schinz, 1821) - Itatiaia, site of PNI (600 m) ( $\sigma^{\pi}$ : PNI 433*), site of PNI (780 m) $\left(\sigma^{x}\right.$ : PNI 1039*). CS: threatened/vulnerable.

Panthera onca (Linnaeus, 1758) - Itatiaia, Retiro de Ramos (Serra das Pyramides) (22²4'S 445'W, 2,180 m) (\# sighting). CS: threatened/ critically endangered.

Puma concolor (Linnaeus, 1771) - Itatiaia, site of PNI (500 m) (I: PNI 386*). CS: threatened/ vulnerable.

Family Mustelidae

Conepatus semistriatus (Boddaert, 1785) Itatiaia, Campos do Itatiaia, Abrigo Rebouças (2,400 m) (according to Ávila-Pires \& Gouvêa (1977), one 
specimen collected but not preserved); Vale do Paraíba (340 m) (* sighted); Último Adeus (no locality data, 700 m) (* visualization). CS: believed threatened.

Eira barbara (Linnaeus, 1748) - Resende, Serrinha do Alambari (»); Itatiaia, site of PNI (550 m) $\left(\sigma^{x}:\right.$ PNI 1203*; $\left.q: 1204^{*}\right)$, lote $90(1,080 \mathrm{~m})$ ( 9 : PNI 83*). CS: believed threatened.

Galictis cuja (Molina, 1782) - Itatiaia, Vale do Paraíba (400 m) (o*: PNI 761*, 778*, 933*, 934*), sight of PNI (830 m) ( $\sigma^{*}$ : PNI 525*; I: PNI 428*), Retiro de Ramos (I: MN 3127).

Lontra longicaudis (Olfers, 1818) - Resende, Serrinha do Alambari ( $\diamond)$; Itatiaia, Vale do Paraíba (400 m) (古: PNI 81*), Mauá (2340’S 46²7’W, 764 m) (»).

Family Procyonidae

Nasua nasua (Linnaeus, 1766) Itatiaia, site of PNI (830 m) ( $0^{x}:$ PNI 1198*), site of PNI (840 m) ( $0^{x}$ : PNI 1217*; $\%$ : PNI 1216*), Maromba (1,100 m) ( $\sigma^{x}$ : PNI 265*), Campos do Itatiaia (Cabeceiras do Rio Petro) (no coordinate data) (\# sighting).

Procyon cancrivorus (G. Cuvier, 1798) Itatiaia, Vale do Paraíba (400 m) ( $0^{x}$ : PNI 348*; Female: PNI 420*, I: PNI 419), site of PNI (830 m) $\left(\sigma^{\pi}: 387^{*}\right)$.

\section{Order Artiodactyla}

Family Tayassuidae

Mazama americana (Erxleben, 1777) - PNI, from lower limit of the forest (* sighting). CS: threatened/endangered.

Pecari tajacu (Linnaeus, 1758) - Resende, Serrinha do Alambari ( $\diamond$ ); Itatiaia, lote 25 (no coordinate data, 900 m) (\$: PNI 1469 - data from voucher cards); Picadão (Engenharia) (no coordinate data, 1,200 m) (I: PNI 145* - same number for a specimen of Tayassu pecari); Maromba (1,300 m) (Female: PNI 1462, 1463), Maromba, lote 27 (900 m) ( $\sigma^{x}$ : PNI 1473); Rio Bonito (no coordinate data, 900 m) (I: PNI 20). CS: threatened/vulnerable.

Tayassu pecari (Link, 1795) - Itatiaia, Rio Bonito (900 m) (I: PNI 24*), Rio Campo Belo, lote 41 (1,150 m) (ơ : PNI 144*, 145 - same number for a specimen of Pecari tajacu), Rio Campo Belo, lot 27 (940 m) (\$: PNI 80*); Maromba (1,000 m) ( $:$ PNI 42 - same number for a specimen of Sciurus aestuans), site of PNI (1,056 m) (\&: AMNH 61536*, $\left.61537^{*}, 61538^{*}\right)$. CS: threatened/endangered.

\section{Order Perissodactyla}

Family Tapiridae

Tapirus terrestris (Linnaeus, 1758) - $*^{*}-$ sighting). CS: threatened/endangered.

\section{Order Rodentia}

Family Sciuridae

Sciurus aestuans Linnaeus, 1766 - Resende, Serrinha do Alambari (»); Itatiaia, site of PNI (830 m) (ơ: PNI 71*, : PNI 725*, 1318); Pinheiro Grande, lote 21 (no coordinate data, $980 \mathrm{~m}$ ) ( $\sigma^{x}:$ PNI 1089*); Maromba (1,100 m) (I: PNI 42* - same number for a specimen of Tayassu pecari, $43 *$ ).

Family Muridae

Nectomys squamipes (Brants, 1827) - Itatiaia, site of PNI (770 m) ( $\sigma^{\star}$ : PNI 1035 - same number for a skull of Rhipidomys mastacalis), Maromba (1,170 m) (\$: HGB-DB 27, 29).

Oligoryzomys flavescens (Waterhouse, 1837) Resende, Serrinha do Alambari (Male: MN 48007); Itatiaia, Maromba (1,170 m) (\$: HGB-DB 34); Campos do Itatiaia (2,300 m) ( $\sigma^{x}$ : MZUSP 2168, 9 : MZUSP 2167).

Oligoryzomys nigripes (Olfers, 1818) Itatiaia, Brejo da Lapa (22 $\left.23^{\circ} \mathrm{S} 44^{\circ} 43^{\prime} \mathrm{W}, 2,100 \mathrm{~m}\right)\left(0^{\star}: \mathrm{MN}\right.$ 48046 \&, 48047 ). Karyotypes: $2 \mathrm{n}=62, \mathrm{FN}=82$. Oryzomys angoya (Hensel, 1873) - Itatiaia, site of PNI ( $830 \mathrm{~m})$ (I: PNI 1031* - same number for a skin of Rhipidomys mastacalis).

Akodon cursor (Winge, 1887) - Resende, Serrinha do Alambari ( $\sigma^{\boldsymbol{x}}$ : MN $47988 \boldsymbol{\bullet}, 47989$ ^, 47993^, 47994^; 9 : MN 47986^, 47987^, 47990^, 47991 *); Itatiaia, Penedo ( $\sigma^{\pi}$ : MZUSP 29223, 29226; 9 : MZUSP 29224); Maromba (1,170 m) (ơ : HGB-DB 21, 22, 23). Karyotypes: 2n = 14, FN = 19/20.

Akodon montensis (Thomas, 1913) - Resende, Serrinha do Alambari (I: HGB-FMH 03); Itatiaia,

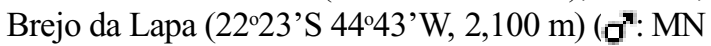

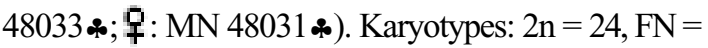
42.

Akodon reigi (Hershkovitz, 1998) - Itatiaia, Brejo da Lapa (2223'S 4443'W, 2,100 m) (o': MN 48041^, 48042^, 48067^, 48070^; + : MN 48029^, 48066 •, 48068 ). Karyotypes: $2 \mathrm{n}=44, \mathrm{FN}=44$. "Akodon" serrensis Thomas, 1902 - Itatiaia, Maromba (1,170 m) (đđ: HGB-DB 28), Brejo da Lapa

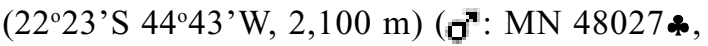


48028 48030 4); Retiro de Ramos (\#) (đđ: MN 2513

holotype of Akodon serrensis leucogula (Miranda Ribeiro, 1905)); Caminho do Couto (22 $22^{\circ}$ 'S $44^{\circ} 42^{\prime} \mathrm{W}$, 2,460 m) (\#); site of PNI (775 m) ( $0^{x}:$ MN 32599$)$. Karyotypes: $2 \mathrm{n}=46, \mathrm{FN}=46$.

Brucepattersonius spp. Hershkovitz, 1998 Itatiaia, Brejo da Lapa (22.23'S 444' $\mathrm{W}, 2,100 \mathrm{~m})\left(\sigma^{\mathrm{x}}\right.$ : MN 48016, 48018, LG109, \&: LG 84, 110, 111, 113, 114), Campos do Itatiaia $(2,300 \mathrm{~m})\left(\sigma^{\boldsymbol{x}}\right.$ : PNI 1191*). CS: believed threatened.

Oxymycterus dasytrichus (Schinz, 1821)-

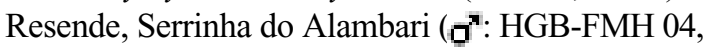
MN 47981 *, 47985; \%: MN 47984, 47982); Itatiaia, site of PNI (815 m) ( $\sigma^{\star}:$ MN $42434 \bullet$, PNI 1205), site of PNI (750 m) (ơ: PNI 1026 - skin; PNI 1029 - skull, both numbers refer to the same individual, PNI 1032*); Itatiaia $\left(22^{\circ} 30^{\prime} \mathrm{S} 44^{\circ} 34^{\prime} \mathrm{W}, 400 \mathrm{~m}\right)$ (I: BMNH $1422316 \diamond)$. Karyotypes: $2 \mathrm{n}=54$. CS: believed threatened.

Oxymycterus sp. - Itatiaia, Alto do Itatiaia

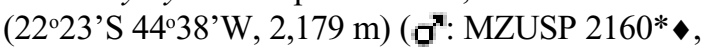
MZUSP 2074; I: AMNH 61542 •, MZUSP 2073);

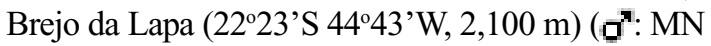
46703). CS: believed threatened.

Thaptomys nigrita (Lichtenstein, 1829) Itatiaia, site of PNI ( $830 \mathrm{~m}$ ) ( ${ }^{x}$ : PNI $865^{*}-$ skin; PNI 851 - skull, both numbers refer to the same indivi-

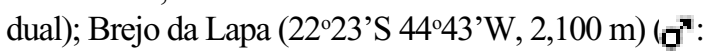
MN 48074); Campos de Itatiaia (2,300 m) ( $\sigma^{\pi}$ : MZUSP 2169*). CS: threatened/vulnerable.

Delomys collinus (Thomas, 1917) - Itatiaia,

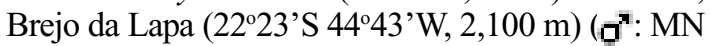
33698, 33700, 44058, 44059^, 44064, 60572, 60575, LG 106; 9 : MN 44062 \&, 44063^, 33701, 33702, 60574, 60576). Karyotypes: $2 \mathrm{n}=82, \mathrm{FN}=86$. CS: believed threatened.

Delomys dorsalis (Hensel, 1872) - Resende, Serrinha do Alambari ( $\sigma^{x}$ : HGB-DB 62*, 64*); Itatiaia, Brejo da Lapa (22 $\left.23^{\prime} \mathrm{S} 44^{\circ} 43^{\prime} \mathrm{W}, 2,100 \mathrm{~m}\right)$ ( $0^{\star}$ : LG 133^, 141 ; ; : LG 138^, 139^, LF 2175);

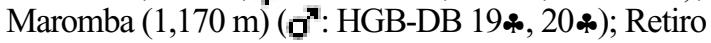
de Ramos (\#), Alto de Itatiaia (22²6'S 4439' W, 2,000 m) ( $\sigma^{x}$ : MZUSP 2163, 2164). Karyotypes: $2 \mathrm{n}=82, \mathrm{FN}$ $=80$.

Juliomys rimofrons Oliveira \& Bonvicino, 2002 - Itatiaia, Brejo da Lapa (2223'S 4443'W, 2100 m) (ơ : MN61646, 46703; ㅇ: MN61647).

Oecomys sp. (Thomas, 1906) - Itatiaia, Hotel Casa Alpina (no coordinate data, 1,600 m) (2 skins, without register number, deposited in the Museum of the Itatiaia National Park, identified as Oecomys simplex); sede do PNI (850 m) (đơ : PNI 1002 - skin; PNI 1006 - skull, both numbers refer to the same individual); Maromba (1,500 m) ( $\sigma^{\pi}$ : PNI 624 - skin; PNI 608* skull, both numbers refer to the same individual).

Rhipidomys sp. (Lund, 1840) Itatiaia, site of PNI (830 m) ( $\sigma^{x}$ : PNI 1031 - skin - same number for a specimen of Oryzomys angoya; PNI 1035 - skull same number for a specimen of Nectomys squamipes, both numbers refer to the same individual). CS: believed threatened.

Family Erethizontidae

Sphiggurus insidiosus (Lichtenstein, 1818) Itatiaia, Maromba $(1,100 \mathrm{~m})\left(\sigma^{x}\right.$ : PNI $186^{*} ; q$ : PNI 240*); Maromba, Picadão (Engenharia) (1,200 m) ( ( PNI 264*). CS: believed threatened.

Family Caviidae

Cavia aperea Erxleben, 1777 - Itatiaia, site of PNI (830 m) ( $\sigma^{x}:$ PNI 404*; I: PNI 630*), Campos do Itatiaia, Abrigo Rebouças (22²2'S 4440’W, 2,300 m) (»).

Family Agoutidae

Agouti paca (Linnaeus, 1766) - Itatiaia, Vale do Paraíba (430 m) (I: PNI 564), site of PNI (830 m) (\$: PNI 241*; I: PNI 242*). CS: threatened/ vulnerable.

Family Hydrochaeridae

Hydrochaeris hydrochaeris (Linnaeus, 1766) Itatiaia, Vale do Paraíba (400 m) (I: PNI 541, 562*), Vale do Paraíba (470 m) (\$: PNI 466*), Vale do Paraíba (500 m) (I: PNI 465*).

Family Echimyidae

Euryzygomatomys spinosus (G Fischer, 1814) Itatiaia, Maromba (1,100 m) (I: MZUSP 8078). CS: believed threatened.

Kannabateomys amblyonyx (Wagner, 1845) Itatiaia, Fazenda Light-Santana das Tocas (no coordinate data, no altitude data) (\$: PNI 1051); Caminho dos Três Picos (lote 126) (22²6'S 44³6'W, 1,200 m) (: PNI 1274). CS: threatened/vulnerable.

Trinomys gratiosus (Moojen, 1948) - Resende, Serrinha do Alambari ( $\sigma^{x}$ : HGB-FMH 05), Maromba $(1170 \mathrm{~m})\left(\sigma^{x}:\right.$ HGB-DB 24), site of PNI (830 m) ( $\sigma^{x}:$ PNI 1025 - skin; PNI 1028 - skull, both numbers refer to the same individual), Penedo (\$: MZUSP 29394).

\section{Order Lagomorpha}

Family Leporidae

Sylvilagus brasiliensis (Linnaeus, 1758)Itatiaia, site of PNI (830 m) ( $0^{x}$ : PNI 422*; Female: 
PNI 48* - same number for a specimen of Philander frenatus, PNI 772*), Vale do Paraíba (400 m) (I: PNI 1503).

The 69 mammal species collected and reported here belong to eight orders and 20 families. Of these, 32 species (46.4\%) are included in the official list of threatened or believed-to-be threatened species of Rio de Janeiro State (Bergallo et al., 2000). Twenty-two species are endemic to the Atlantic Forest (Fonseca et al., 1996; Oliveira, 1998; Lara \& Patton, 2000; Silva, 2001; Geise et al., 2001), and $31.9 \%$ of these are endemic.

The orders Rodentia, Carnivora, and Didelphimorphia had the highest species richness, with 25 $(36.2 \%), 14(20.3 \%)$, and 13 species $(18.9 \%)$, respectively. Those orders with less species were Artiodactyla, with three (4.3\%) and Perissodactyla and Lagomorpha, both with one species (1.4\%), the last of which is a monospecific order in South America.

Fifty-one species (74\%) were small mammals. (The biggest mammal presently registered for this area is Tapirus terrestris.) The smallest of these were rodents (genera Thaptomys, Oligoryzomys, and Juliomys) and marsupials (genera Monodelphis and Marmosops).

When species were grouped according to vegetation, 16 species occurred in the Floresta SubMontana; 56, in the Floresta Montana; five, in the Floresta Alto-Montana; and 21, in the Campos de Altitude. Some species occurred in more than one vegetation type, and others occurred in only one. Of the 56 species recorded for the Floresta Montana, 31 were registered only in this vegetation; from the two species listed for the Floresta Alto-Montana, only one species occurred only there; and eight species were specific to the Campos de Altitude (Table 1), of which six species belongs to the subFamily Sigmodontinae.

Figs. 1, 2, and 3 show that the communities of marsupials, primates, and rodents formed an altituderelated continuum. Marsupials were mainly recorded between 700 and $900 \mathrm{~m}$ a.s.l. while only five species were observed above $1,000 \mathrm{~m}$ (Fig. 1). Primates were recorded in greater number between the lower level of the park and 1,300 $\mathrm{m}$ a.s.l., but none were observed above 1,700 m a.s.l. (Fig. 2). Rodents showed a clear distributional pattern along the altitude gradient, varying from those restricted to lower altitudes to those restricted to higher ones (Fig. 3). Species richness was higher between $800 \mathrm{~m}$ and 1,100 $\mathrm{m}$ a.s.l. (Fig. 3). However, as species data in this study were not collected with identical methodologies along the altitude gradient, the results must be analyzed with care.

\section{DISCUSSION}

The number of species recorded for the Itatiaia massif is similar to the first list for the whole of Rio de Janeiro State (Tribe, 1987). An important study about the mammal fauna of the PNI was carried out by Ávila-Pires \& Gouvêa (1977) who listed 62 mammals, of which 47 are nonvolant species. Faunal survey scarcity, taxonomic problems, and difficulties in collecting some orders, explain present lack of knowledge of most Atlantic Forest mammal fauna. However, increasing numbers of specialists and more collecting in the area of PNI and its surroundings have increased knowledge of the local mammal fauna. Consequently, the species listed by Ávila-Pires \& Gouvêa (1977) increased by 22, which may also be explained by the increase of taxonomic characters currently used (e.g., karyotypes and DNA sequences), as well as rectification of misidentifications of some species. This difference might have been greater if genera with morphologically similar species (e.g., Brucepattersonius, Smith \& Patton, 1999) had been systematically reviewed, allowing a formal species description through genetic techniques, as has been already done with Delomys, now recognized through karyological data to have three different species (Bonvicino \& Geise, 1995; Voss, 1993).

Some species not listed by us probably occur in the area, such as Mazana nana (Artiodactyla) and Potos flavus (Carnivora), as the PNI is included in the area of their geographic distribution (Eisenberg \& Redford, 1999). In the public exhibition of the PNI Museum some specimens of both species are found. While lacking collection data, local people say that these were captured in the park.

A fauna was observed that was peculiar to the Campos de Altitude. Included species (mainly Sigmodontinae rodents) are also observed in other localities with similar habitats, e.g., "Akodon" serrensis, Akodon reigi, and Delomys collinus, found in the National Park of Bocaina, Caparaó, and Serra dos Órgãos (Bonvicino et al., 1997; L. Geise, pers. obs.). Such allopatric distributions can probably be 
explained by isolation in montane rainforest remnants during climatic dry periods (Montane Isolate Hypothesis, Moreau (1966)) and, if we consider the low divergence rates observed in samples of Akodon montensis between such montane isolates (Geise et al., 2001), they have not resulted in significant phenotypic evolution or speciation, maintaining until now the same species. The Montane Isolates Hypothesis, first introduced by Moureau (1966) and postulating that isolation in montane rainforest remnants during climatic dry periods led to divergence and speciation, has not been explicitly examined in the Atlantic Forest, even though the mountainous topography of the region suggests that montane isolation is a plausible mechanism for geographic isolation. For these taxa, montane isolation almost certainly holds, but it is not clear whether it will continue to drive future speciation. Again, future analyses of DNA variation in small mammals will be useful. Species richness in PNI and its surroundings was higher in Floresta Montana between 500 and 1,500 $\mathrm{m}$ a.s.l. with a mid-elevation richness peak. Although it is postulated that species richness is higher in lowland tropical forest (MacArthur, 1972; Patterson et al., 1996, 1998), recent studies have showed that species richness can reach its maximum in mid-elevations, yielding a curvilinear pattern along elevational gradients (e.g., for birds, Goodman et al., 1996; for small mammals, Heaney, 2001). Hypotheses explaining elevational changes in species richness vary (Brown, 2001; Rahbek, 1995). Heaney (2001), studying small mammal elevational gradients in the Philippines, suggested that annual rainfall, total abundance of individuals in the community, food resource diversity, areas of reduced competition from other organisms, areas characterized by high rates of speciation, and areas of community overlap were correlated with diversity.

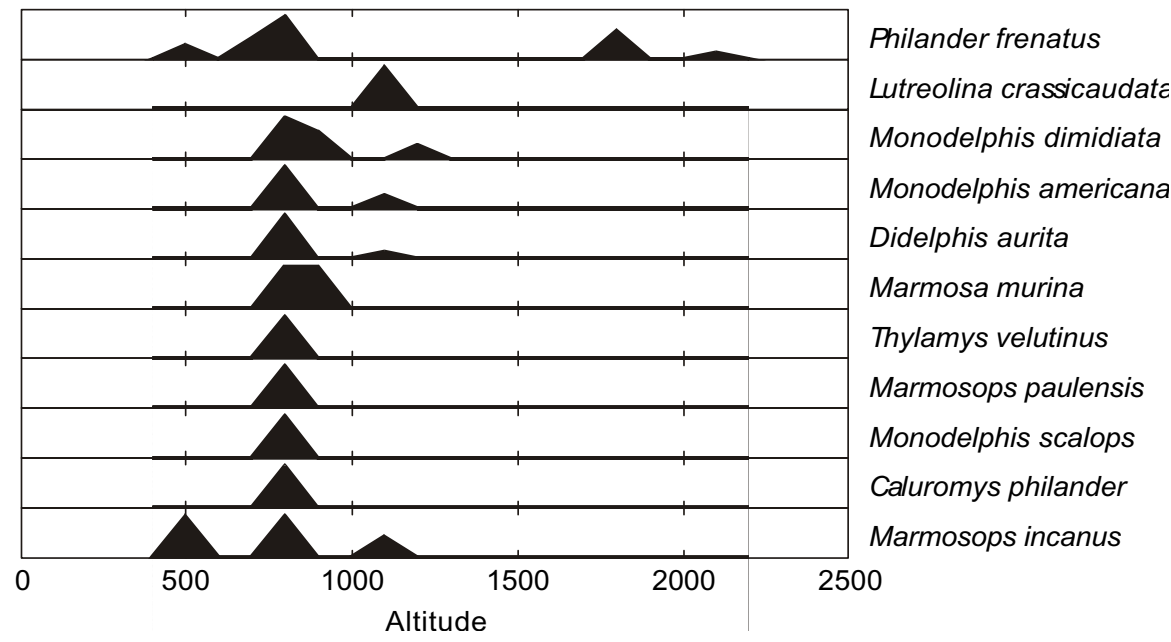

Fig. 1 - Distribution of the relative frequency of capture of marsupial species in Itatiaia massif, along an altitudinal axis.

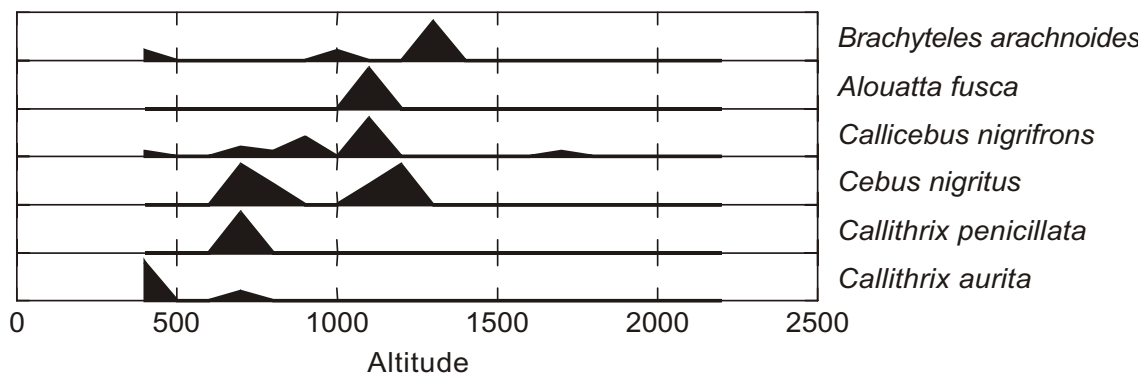

Fig. 2 - Distribution of the relative frequency of capture of primate species in Itatiaia massif, along an altitudinal axis. 
TABLE 1

Order and species reported for the Itatiaia National Park and its surroundings in different vegetation types. Filled cells indicate the species presence in that vegetation type.

\begin{tabular}{|c|c|c|c|c|}
\hline Species & Submontana & Montana & Alto-Montana & Campos de Altitude \\
\hline \multicolumn{5}{|l|}{ Caluromys philander } \\
\hline \multicolumn{5}{|l|}{ Didelphis aurita } \\
\hline \multicolumn{5}{|c|}{ Lutreolina crassicaudata } \\
\hline \multicolumn{5}{|l|}{ Marmosa murina } \\
\hline \multicolumn{5}{|l|}{ Marmosops paulensis } \\
\hline \multicolumn{5}{|l|}{ M. incanus } \\
\hline \multicolumn{5}{|l|}{ Micoureus travassosi } \\
\hline \multicolumn{5}{|c|}{ Monodelphis americana } \\
\hline \multicolumn{5}{|l|}{ M. dimidiata } \\
\hline \multicolumn{5}{|l|}{ M. scalops } \\
\hline \multicolumn{5}{|l|}{ Philander frenatus } \\
\hline \multicolumn{5}{|l|}{ Thylamys velutinus } \\
\hline \multicolumn{5}{|l|}{ Bradypus variegatus } \\
\hline \multicolumn{5}{|l|}{ Cabassous tatouay } \\
\hline \multicolumn{5}{|l|}{ Dasypus novemcinctus } \\
\hline \multicolumn{5}{|l|}{ D. septemcinctus } \\
\hline \multicolumn{5}{|c|}{ Myrmecophaga tridactyla } \\
\hline \multicolumn{5}{|l|}{ Tamandua tetradactyla } \\
\hline \multicolumn{5}{|l|}{ Callithrix aurita } \\
\hline \multicolumn{5}{|l|}{ C. penicillata } \\
\hline \multicolumn{5}{|l|}{ Alouatta fusca } \\
\hline \multicolumn{5}{|c|}{ Brachyteles arachnoides } \\
\hline \multicolumn{5}{|l|}{ Callicebus nigrifrons } \\
\hline \multicolumn{5}{|l|}{ Cebus nigritus } \\
\hline \multicolumn{5}{|l|}{ Cerdocyon thous } \\
\hline \multicolumn{5}{|c|}{ Chrysocyon brachyurus } \\
\hline \multicolumn{5}{|c|}{ Herpailurus yaguarondi } \\
\hline Leopardus pardalis & & & & \\
\hline L. tigrinus & & & & \\
\hline L. wiedii & & & & \\
\hline Panthera onca & & & & \\
\hline Puma concolor & & & & \\
\hline Conepatus semistriat & & & & \\
\hline Eira barbara & & & & \\
\hline Galictis cuja & & & & \\
\hline Lontra longicaudis & & & & \\
\hline Nasua nasua & & & & \\
\hline Procyon cancrivorus & & & & \\
\hline Pecari tajacu & & & & \\
\hline
\end{tabular}


TABLE 1 (Continued).

\begin{tabular}{|c|c|c|c|c|}
\hline Species & Submontana & Montana & Alto-Montana & Campos de Altitude \\
\hline Tayassu pecari & & & & \\
\hline Sciureus aestuans & & & & \\
\hline Nectomys squamipes & & & & \\
\hline Oligoryzomys flavescens & & & & \\
\hline O. nigripes & & & & \\
\hline Oryzomys angoya & & & & \\
\hline Akodon cursor & & & & \\
\hline A. montensis & & & & \\
\hline Akodon reigi & & & & \\
\hline "Akodon" serrensis & & & & \\
\hline Brucepattersonius spp. & & & & \\
\hline Oxymycterus dasytrichus & & & & \\
\hline Oxymycterus sp. & & & & \\
\hline Thaptomys nigrita & & & & \\
\hline Delomys collinus & & & & \\
\hline D. dorsalis & & & & \\
\hline Juliomys rimofrons & & & & \\
\hline Oecomys sp. & & & & \\
\hline Rhipidomys sp. & & & & \\
\hline Sphiggurus insidiosus & & & & \\
\hline Cavia aperea & & & & \\
\hline Agouti paca & & & & \\
\hline Hydrochaeris hydrochaeris & & & & \\
\hline Euryzygomatomys spinosus & & & & \\
\hline Kannabateomys amblyonyx & & & & \\
\hline Trinomys gratiosus & & & & \\
\hline Sylvilagus brasiliensis & & & & \\
\hline
\end{tabular}

Although the terms Floresta Submontana, Floresta Montana, Floresta Alto-Montana and Campos de Altitude are useful for descriptive purposes, what actually exists is a continuum between them. The three orders studied here showed a continuum pattern related to the altitude gradient. The majority of marsupials presented an optimum between 500 and 1,000 m, forming a distribution with few intermediate species. On the other hand, the rodent community showed all kinds of species distributions, including those with wide distribution and those restricted to a single altitude band. The Order Primates formed an altitude- related continuum pattern, but none of the species were observed above $1,720 \mathrm{~m}$ a.s.l. Grelle (2000) observed a 1,500 m maximum limit for endemic primate species from the Atlantic Forest. According to Grelle (2000), the absence of primates in high altitudes there may be due to the tree-line lowering during the glaciations (Safford, 1999). This also explains why only cursorial mammals were found in Campos de Altitude. The low diversity of Didelphimorphia in this area of the PNI shows the necessity of a major sampling effort, requiring especially the use of pitfall traps, since such fauna have already been collected in other Campos de Altitude (L. Geise, pers. obs.; Bonvicino et al., 1997). 
A higher richness was observed in the Floresta Montana and the Campos de Altitude. Both Submontana and Alto-Montana areas showed a lower richness, probably due to the fact that the former has a narrow altitude band ( 400 to $500 \mathrm{~m}$ ) in Itatiaia and fewer samples were collected in both bands (authors, pers. obs.). This could be observed due to the occurrence of some species in all bands except in Alto Montana (e.g., Cerdocyon thous, Leopardus tigrinus, Galictis cuja, Conepatus semistriatus and Lontra longicaudis). Although specimens of Oligoryzomys nigripes were only collected in Campos de Altitu$d e$, and specimens of Philander frenatus were not collected in the submontana region, it is possible that they are present in all vegetational bands since these species can be found at sea level in other regions (e.g., Pereira et al., 2001).

An Akodontini domain was observed in the Campos de Altitude, as half of the Sigmodontinae rodents are from this Tribe. The same pattern was mentioned by Bonvicino et al. (1997) for Caparaó National Park. In the Akodon genus, a species segregation occurs, since $A$. cursor is found at low altitudes, "Akodon" montensis and A. serrensis at mid- and high altitudes, and Akodon reigi only in high altitudes.

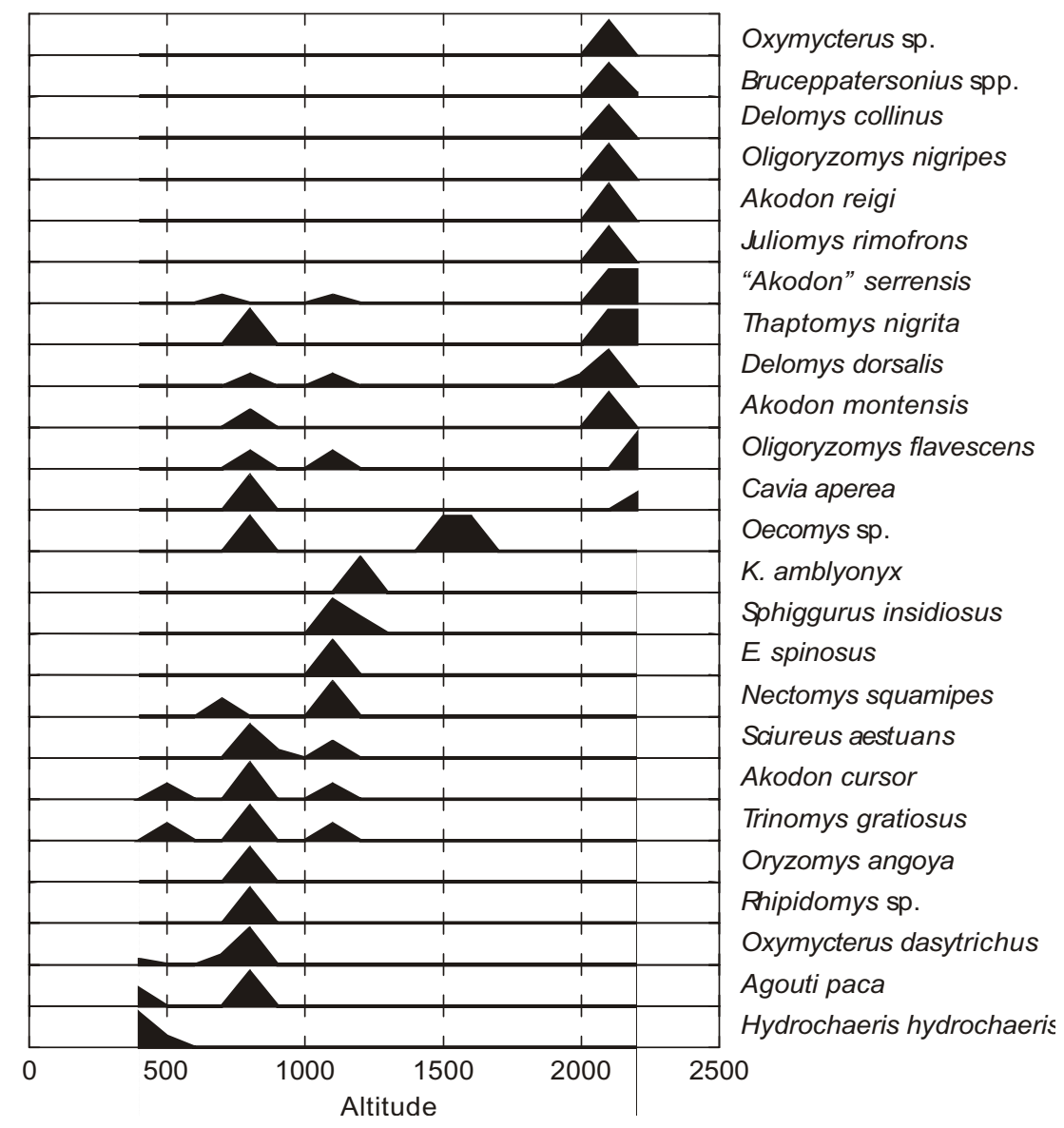

Fig. 3 - Distribution of the relative frequency of capture of rodent species in Itatiaia massif, along an altitudinal axis. Table 1 Order and species reported for the Itatiaia National Park and its surroundings in different vegetation types. Filled cells indicate the species presence in that vegetation type. 
Forest fragments in Rio de Janeiro State have been drastically reduced in recent decades (TanizakiFonseca \& Moulton, 2000), with no records of original diversity. The peculiarities of the PNI region and constant loss of its vegetation cover by anthropic pressures, such as fire, indicate the need to increase understanding of its biota, through such measures as long-term research programs with mammals, including species of Chiroptera. Such programs, including sporadic captures as well as correct species identification through morphological analyses and using genetic techniques, are necessary to adequately assign correct diversity status in this important area. It is also essential to conserve and protect the pristine Atlantic Forest vegetation as well as the Campos de Altitude ecosystem, where most cases of endemism in the PNI are found. Research on other groups of animals and plants must be promoted as it will improve identification of evolutionarily distinct regions and important ecological attributes so as to develop more informed conservation efforts.

Acknowledgements - We thank P. Volpato, W. London, and G. London, from Serrinha do Alambari, and the Chief of PNI, L. Nascimento, for local support. LG and HGB received research grants from CNPq (LG - Process n. 466093/00-4, HGB - Process N. 462003/00-0) and research fellowships from CNPq; LGP received a technical fellowship from FAPERJ and a graduate fellowship from CNPq; DEPB received a graduate fellowship from FAPESP. This work was partially supported by PROBIO/MMA/ CNPq/BIRD and PRONEX/CNPq. We thank B. Lemos and M. Weksler for specimen identification in the Museum of Comparative Zoology (Harvard) and the American Museum of Natural History. We are also grateful to Dr. J. Creed for the English revision.

\section{REFERENCES}

AURICCHIO, P., 1995, Primatas do Brasil. Projeto Editorial UnG. Terra Brasilis Editora Ltda, São Paulo, 168p.

ÁVILA-PIRES, F. D. \& GOUVÊA, E., 1977, Mamíferos do Parque Nacional do Itatiaia. Bol. Mus. Nac., 291: 1-29.

BERGALLO, H. G, GEISE, L., BONVICINO, C. R., CERQUEIRA, R., D'ANDREA, P., ESBERÁRD, C. E., FERNANDEZ, F., GRELLE, C. E., PERACCHI, A., SICILIANO, S. \& VAZ, S. M., 2000, Mamíferos, pp. 125-135. In: H. G. Bergallo, C. F. D. Rocha, M. A. S. Alves \& M. Van Sluys, (orgs.), A fauna ameaçada de extinção do Estado do Rio de Janeiro. EDUERJ, Rio de Janeiro, 166p.

BONVICINO, C. R., LANGGUTH, A., LINDBERGH, S. M. \& DE PAULA, A. C., 1997, An elevational gradient study of small mammals at Caparaó National Park, Southeastern Brazil. Mammalia, 61(4): 547-560.
BONVICINO, C. R. \& GEISE, L., 1995, Taxonomic status of Delomys dorsalis collinus Thomas, 1917 (Rodentia, Cricetidae) and description of a new karyotype. Z. Säuget., 60: $124-127$

BROWN, J. H., 2001, Mammals on mountainsides: elevational patterns of diversity. Global Ecol. Biogeogr., 10: 101-109.

BUENO, C., 1998, Conservação de biodiversidade nos parques urbanos: caso do Parque Nacional da Tijuca. Dissertação de Mestrado, UNESA, 156p.

CONSERVATION INTERNATIONAL OF BRAZIL, FUNDAÇÃO SOS MATA ATLÂNTICA, FUNDAÇÃO BIODIVERSITAS, INSTITUTO DE PESQUISAS ECOLÓGICAS, SECRETARIA DO MEIO AMBIENTE DO ESTADO DE SÃO PAULO (SEMAD) \& INSTITUTO ESTADUAL DE FLORESTAS-MG, 2000, Avaliação e ações prioritárias para a conservacão da biodiversidade da Mata Atlântica e Campos Sulinos. MMA/SBF, Brasília, 1-40.

EISENBERG, J. F. \& REDFORD, K. H., 1999, Mammals of the neotropics. Vol. 3. University of Chicago Press, Chicago and London, 609p.

EMMONS, L. H. \& FEER, F., 1997, Neotropical rainforest mammals. A field guide. 2. ed., University of Chicago Press, Chicago and London.

FLEMING, T. H., 1979, Life-history strategies, pp. 1-61. In: D. M. Stoddart (ed.), Ecology of small mammals. Chapman and Hall, London.

FONSECA, G. A. B., HERMAN, G., LEITE, Y. L., MITTERMEIER, R., RYLANDS, A. B. \& PATTON, J. L., 1996, Lista anotada dos mamíferos do Brasil. Occ. Pap. Conserv. Biol., Washington, DC, 4: 1-38.

FORD, C. E. \& HAMERTON, J. L., 1956, A colchicine hypotonic citrate squash sequence for mammalian choromosome. Stain Technology, Baltimore, 31: 247-251.

GAUCH-JR, H. G., 1982, Multivariate analysis in community ecology. Cambridge Studies in Ecology, Cambridge University Press, Cambridge, 314p.

GEISE, L., 1995, Os roedores Sigmodontinae (Rodentia, Muridae) do Estado do Rio de Janeiro. Sistemática, citogenética, distribuição e variação geográfica. Tese de Doutorado, UFRJ, 388p.

GEISE, L., CANAVEZ, F. C. \& SEUÁNEZ, H. N., 1998, Comparative karyology in Akodon (Rodentia, Sigmodontinae) from Southeastern Brazil. J. Her., Cary, 89: 158-163.

GEISE, L., SMITH, M. F. \& PATTON, J. L., 2001, Diversification in the genus Akodon (Rodentia, Sigmodontinae) in Southeastern South America: Mitochondrial DNA sequences Analysis. Journal of Mammalogy, 82(1): 92-101.

GOODMAN, S. M., ANDRIANARIMISA, A., OLSON, L. E. \& SORIMALALA, V., 1996, Patterns of elevational distribution of birds and small mammals in the humid forests of Montagne D'Ambre, Madagascar. Ecotropica, 2: 87-98. 
GRELlE, C. E. de V., 2000, Aerografia dos primatas endêmicos da Mata Atlântica. Tese de Doutorado, UFRJ$\mathrm{MN}, 150 \mathrm{p}$.

HEANEY, L. R., 2001, Small mammal diversity along elevational gradients in the Philippines: an assessment of patterns and hypotheses. Global Ecol. Biogeogr., 10: 15-39.

HOLT, E. G, 1928, Ornithology of Serra do Itatiaya, Braz. Bull. Amer. Mus. Nat. Hist., 57: 251-326.

LANGE, R. B. \& JABLONSKI, E. F. (eds.), 1998, Mammalia do Estado do Paraná - Marsupialia. Estudos de Biologia, vol. 43. Sociedade Paranaense de Cultura - Editora Universitária Champagnat, Curitiba, 224p.

LARA, M. C. \& PATTON, J. L., 2000, Evolutionary diversification of spiny rats (genus Trinomys, Rodentia: Echimyidae) in the Atlantic Forest of Brazil. Zool. J. Linn. Soc., London, 130: 661-686.

MaCARTHUR, R. H., 1972, Geographical ecology: patterns in the distribution of species. Harper \& Row Publishers, New York.

MIRANDA-RIBEIRO, A., 1905, Vertebrados do Itatiaia. Arch. Mus. Nac., 13: 163-190.

MOREAU, R. E., 1966, The bird faunas of Africa and its islands. Academic Press, New York.

MUSTRANGI, M. A. \& PATTON, J. L., 1997, Phylogeography and systematics of the Slender Mouse Opossum Marmosops (Marsupialia, Didelphidae). University of California Press, Berkeley, Zoology, vol. 130, 86p.

OLIVEIRA, J. A., 1998, Morphometric assessment of species groups in the South American rodent genus Oxymycterus. Doctor of Philosophy Thesis, Texas Tech University, Lubbock, Texas, 320p.

OLIVEIRA, J. A. \& BONVICINO, C. R., 2002, A new species of sigmodontine rodent from the Atlantic forest of eastern Brazil. A. Theriol., 47: 307-322.

PATTERSON, B. D., PACHECO, V. \& SOLARI, S., 1996, Distributions of bats along an elevational gradient in the Andes of south-eastern Peru. J. Zool., London, 240: 637-658.

PATTERSON, B. D., STOTZ, D. F., SOLARI, S., FITZPATRICK, J. W. \& PACHECO, V., 1998, Contrasting patterns of elevational zonation for birds and mammals in the Andes of southeastern Peru. J. Biogeogr., 25: 593-607.

PEREIRA, L. G., TORRES, S. E. M., SILVA, H. S. \& GEISE, L., 2001, Non-volant mammals of Ilha Grande and adjacent areas in southern Rio de Janeiro, Brazil. Bol. Mus. Nac., N. S., Zool., 459: 1-15.

PINTO, O., 1951, Aves do Itatiaia: lista remissiva e novas achegas à avifauna da região. Pap. Dep. Zool. S. Paulo, 10(9): 155-208.

PINTO, O., 1954, Aves do Itatiaia. Bol. Pque. Nac. Itatiaia, 3 : 1-87.

RAHBEK, C., 1995, The elevational gradient of species richness: a uniform pattern? Ecography, 18: 200-205.

SAFFORD, H. D., 1999, Brazilian Páramos I. An introduction to the physical enviroment and vegetation of the campos de altitude. J. Biogeogr., 26: 693-712.

SEGADAS-VIANA, F. \& DAU, L., 1965, Ecology of the Itatiaia range, southeastern Brazil. II- Climates and altitudinal climatic zonation. Arq. Mus. Nac., 53: 3153.

SMITH, M. F. \& PATTON, J. L., 1999, Phylogenetic relationships and the radiation of sigmodontine rodents in South America: Evidence from cytochrome b. J. Mamm. Evol., 6 (2): 89-128.

SILVA, H. S., 2001, Padrões de distribuição de três espécies de roedores neotropicais do gênero Akodon (Rodentia: Sigmodontinae). Monografia de Bacharelado, UERJ, 49p.

SILVA JR., J. S., 2001, Especiação nos macacos prego e caiararas, Gênero Cebus Erxleben, 1777 (Primates, Cebidae). Rio de Janeiro. Tese de Doutorado, Universidade Federal do Rio de Janeiro, 377p.

TANIZAKI-FONSECA, K. \& MOULTON, T. P., 2000, A fragmentação da Mata Atlântica no Estado do Rio de Janeiro e a perda de diversidade. In: H. G. Bergallo, C. F. D. Rocha, M. A. S. Alves \& M. Van Sluys (orgs.), A fauna ameaçada de extinção do Estado do Rio de Janeiro. EDUERJ, Rio de Janeiro, 166p.

TER BRAAK, C. J. F. \& LOOMAN, C. W. N., 1995, Regression, pp. 29-77. In: R. H. G. Jongman, C. J. F. ter Braak \& O. F. R. Van Tongeren (eds.), Data analysis in community and landscape ecology. Cambridge University Press, Cambridge.

TRIBE, C. J., 1987, A mastofauna do Estado do Rio de Janeiro, com especial referência à ordem Polyprotodontia (Marsupiais). Dissertação de Mestrado, Universidade Federal do Rio de Janeiro, Museu Nacional, 244p.

TRIBE, C. J., 1996, The Neotropical rodent genus Rhipidomys (Cricetidae: Sigmodontinae) - a taxonomic revision. Doctor of Philosophy Thesis, University College London, London, $316 \mathrm{p}$.

ULE, E., 1896, Relatório de uma excursão botânica feita na Serra do Itatiaia. Rev. Mus. Nac., 9: 185-223.

UNITED STATES BOARD ON GEOGRAPHICAL NAMES. BRASIL, 1963, Official Standard Names. Office of Geography, Department of Interior, Washington, DC, 915p.

URURAHY, J. C. C., COLLARES, J. E. R., SANTOS, M. M. \& BARRETO, R. A. A., 1983, Folhas SF.23/24 Rio de Janeiro/ Vitória; geologia, geomorfologia, pedologia, vegetação e uso potencial da terra. In: Projeto RADAMBRASIL, As regiões fitoecológicas, sua natureza e seus recursos econômicos. Estudo fitogeográfico. Rio de Janeiro, 780p. (4 - Vegetação).

VIVO, M., 1991, Taxonomia de Callithrix Erxleben, 1777 (Callithrichidae, Primates). Fundação Biodiversitas, Belo Horizonte, $105 \mathrm{p}$.

VOSS, R. S., 1993, A revision of the Brasilian Muroid Rodent Genus Delomys with remarks on "Thomasomyine" Characteres. Am. Mus. Novitates, 3073: 1-44.

WEKSLER, M., 1996, Revisão sistemática do grupo de espécies nitidus do gênero Oryzomys (Rodentia: Sigmodontinae). Dissertação de Mestrado, Universidade Federal do Rio de Janeiro, Museu Nacional, 210p.

WILSON, D. E. \& REEDER, D. A. (eds.), 1993, Mammal species of the world. A taxonomic and geographic reference. $2^{\text {nd }}$ ed., Smithsonian Institution Press, Washington and London. 